\title{
Genomic and bioinformatics analysis of human adenovirus type 37: New insights into corneal tropism Christopher M Robinson ${ }^{1,3}$, Fatemeh Shariati1,2, Allison F Gillaspy ${ }^{3,5}$, David W Dyer ${ }^{3,5}$ and James Chodosh*1,2,3,4
}

\begin{abstract}
Address: ${ }^{1}$ Molecular Pathogenesis of Eye Infection Research Center, Dean A. McGee Eye Institute, 608 Stanton L. Young Blvd., Oklahoma City, OK 73104, USA, 2Department of Ophthalmology, University of Oklahoma Health Sciences Center, 1100 North Lindsay, Oklahoma City, OK 73104, USA, ${ }^{3}$ Department of Microbiology \& Immunology, University of Oklahoma Health Sciences Center, 1100 North Lindsay, Oklahoma City, OK 73104, USA, ${ }^{4}$ Department of Cell Biology, University of Oklahoma Health Sciences Center, 1100 North Lindsay, Oklahoma City, OK 73104, USA and ${ }^{5}$ Laboratory for Genomics and Bioinformatics, University of Oklahoma Health Sciences Center, 1100 North Lindsay, Oklahoma City, OK
\end{abstract} 73104, USA

Email: Christopher M Robinson - chris-robinson@ouhsc.edu; Fatemeh Shariati - fatemeh-shariati@ouhsc.edu; Allison F Gillaspy - allisongillaspy@ouhsc.edu; David W Dyer - david-dyer@ouhsc.edu; James Chodosh* - james-chodosh@ouhsc.edu

* Corresponding author

Published: 9 May 2008

BMC Genomics 2008, 9:213 doi:10.1186/147|-2164-9-213
Received: 7 December 2007

Accepted: 9 May 2008

This article is available from: http://www.biomedcentral.com/I47|-2/64/9/2/3

(C) 2008 Robinson et al; licensee BioMed Central Ltd.

This is an Open Access article distributed under the terms of the Creative Commons Attribution License (http://creativecommons.org/licenses/by/2.0), which permits unrestricted use, distribution, and reproduction in any medium, provided the original work is properly cited.

\begin{abstract}
Background: Human adenovirus type 37 (HAdV-37) is a major etiologic agent of epidemic keratoconjunctivitis, a common and severe eye infection associated with long-term visual morbidity due to persistent corneal inflammation. While HAdV-37 has been known for over 20 years as an important cause, the complete genome sequence of this serotype has yet to be reported. A detailed bioinformatics analysis of the genome sequence of HAdV-37 is extremely important to understanding its unique pathogenicity in the eye.
\end{abstract}

Results: We sequenced and annotated the complete genome of HAdV-37, and performed genomic and bioinformatics comparisons with other HAdVs to identify differences that might underlie the unique corneal tropism of HAdV-37. Global pairwise genome alignment with HAdV9 , a human species $D$ adenovirus not associated with corneal infection, revealed areas of nonconserved sequence principally in genes for the virus fiber (site of host cell binding), penton (host cell internalization signal), hexon (principal viral capsid structural protein), and E3 (site of several genes that mediate evasion of the host immune system). Phylogenetic analysis revealed close similarities between predicted proteins from HAdV-37 of species $D$ and HAdVs from species $B$ and E. However, virtual $2 D$ gel analyses of predicted viral proteins uncovered unexpected differences in $\mathrm{pl}$ and/or size of specific proteins thought to be highly similar by phylogenetics.

Conclusion: This genomic and bioinformatics analysis of the HAdV-37 genome provides a valuable tool for understanding the corneal tropism of this clinically important virus. Although disparities between HAdV-37 and other HAdV within species $D$ in genes encoding structural and host receptor-binding proteins were to some extent expected, differences in the E3 region suggest as yet unknown roles for this area of the genome. The whole genome comparisons and virtual $2 \mathrm{D}$ gel analyses reported herein suggest potent areas for future studies. 


\section{Background}

Adenoviruses (AdV) in the Adenoviridae family have been divided into four genera: Mastadenovirus, Aviadenovirus, Atadenovirus, and Siadenovirus [1]. The AdV was first isolated from human adenoids and characterized by two different research teams [2,3]. Human AdV (HAdV) fall within the genus Mastadenovirus, and cause a wide array of diseases including acute respiratory disease, gastroenteritis, and ocular surface infection [4-6]. The AdV is nonenveloped with a double stranded linear genome that ranges from 26 to $45 \mathrm{~kb}$ in size. The icosahedral shaped capsid ranges from 70 to 100 nanometers in diameter [7]. There are 51 known HAdV serotypes classified into 6 species (A-F), based on restriction enzyme analysis and hemaglutination assays, later confirmed by genome analyses and phylogenetic calculations. Recently, a proposed fifty-second HAdV serotype was identified and placed into a new species $G[8]$.

HAdV-37 was originally isolated in 1976 from 62 eyes and 9 genitourinary sites, and subsequently characterized as a new serotype in 1981 [9]. HAdV-37 is a major etiologic agent of epidemic keratoconjunctivitis, an explosive and highly contagious infection of the conjunctiva and cornea, and continues to cause outbreaks [10]. HAdV-37 also was recently implicated in the pathogenesis of obesity [11].

Although HAdV species D contains the most serotypes, complete sequence is available for only 6 - HAdV-9, HAdV-17, HAdV-26, HAdV-46, HAdV-48, and HAdV-49 and none of these have been associated with epidemic keratoconjunctivitis. In this study, we have sequenced the complete genome of HAdV-37 and describe its overall organization. The HAdV-37 genome appears in most respects typical of other HAdV. However, global pairwise genome alignments, phylogenetic analyses, and in silico comparisons of putative viral proteins revealed unique characteristics of the genome, including areas of non-conserved sequence in the penton, hexon, E3, and fiber regions, and differences in size and/or pI of select predicted HAdV-37 proteins. Understanding the disparities between the HAdV-37 genome and those of species D HAdV with dissimilar tissue tropisms may lead to improved understanding of the genomic determinants of infection.

\section{Results}

\section{General features}

The genome length of HAdV-37 was found to be 35,213 base pairs with a base composition of $22.8 \% \mathrm{~A}, 20.6 \% \mathrm{~T}$, $28.3 \% \mathrm{C}, 28.3 \% \mathrm{G}$. The $56.6 \% \mathrm{GC}$ content is on the lower end of the $57-59 \%$ range previously reported for HAdVs within species D [7]. CpG dinucleotide analysis of HAdV37 performed using FUZZNUC software [12] revealed
2389 CpG dinucleotides located within the genome (data not shown). We identified the predicted 4 early, 2 intermediate, and 5 late transcription regions similar to those described in other completely sequenced HAdVs (Figure 1 ), including 35 predicted coding sequences within the HAdV-37 genome and 8 hypothetical ORFs.

The 5' and 3' termini of the HAdV genome are composed of inverted terminal repeat (ITR) sequences which for HAdV-37 were determined to be 159 bp in length. These sites serve as replication origins for the virus [7]. The motif located at the extreme termini of the HAdV-37 genome consists of a CATCATCATAAT, which is unique among previously sequenced HAdV serotypes. Unique sequences for the extreme termini have been also observed in other HAdVs including HAdV-4 [13]. The conserved ATAATATACC motif within the ITR, which interacts with the terminal protein precursor (pTP) and polymerase complex during DNA replication [14], was determined at base pairs 8-17. A NFIII/Oct-1 recognition site (TATGCAAAT) was identified within the ITR of HAdV-37 at nucleotides 40-48. A Sp1 binding site (GGGGCGGA) was identified at nucleotides 73-80. Also, a NFI/CTFI (TGGGGCGGAGCCA) site was located at overlapping nucleotides 72-84.

\section{Global pairwise alignment}

The mVISTA Limited Area Global Alignment of Nucleotides (LAGAN) tool was used to align and compare paired viral sequences [15]. We compared genomic sequence correspondence across the whole genome of HAdV-37 to representative HAdV serotypes from each of the six HAdV species. Comparison of the HAdV-37 genome with that of HAdV-9, also within species D, showed a much higher degree of conservation than with representative HAdVs from other species, but demonstrated disparity in the penton, hexon, E3, and fiber regions (Figure 2).

\section{Early genes}

E1A is the first transcriptional unit to be expressed during infection [7]. A common RNA from this region is the source of several alternatively spliced E1A transcripts [16]. The E1A proteins regulate the transcription of viral and cellular genes $[17,18]$. Based on splice donor and acceptor sites, two putative proteins of 253 and 191 amino acids with corresponding molecular weights of $28.2 \mathrm{kDa}$ and $21.2 \mathrm{kDa}$, respectively, were identified in the HAdV-37 genome (Table 1). The HAdV-37 E1A $21.2 \mathrm{kDa}$ protein is $89 \%$ identical and $96 \%$ similar to the HAdV-9 homologue (Table 2). A protein corresponding to the previously predicted 10S protein from previous studies of HAdVs was not identified in our analysis. The predicted TATA box was identified at nucleotide 477 and the polyadenylation signal predicted to be at position 1451 . 


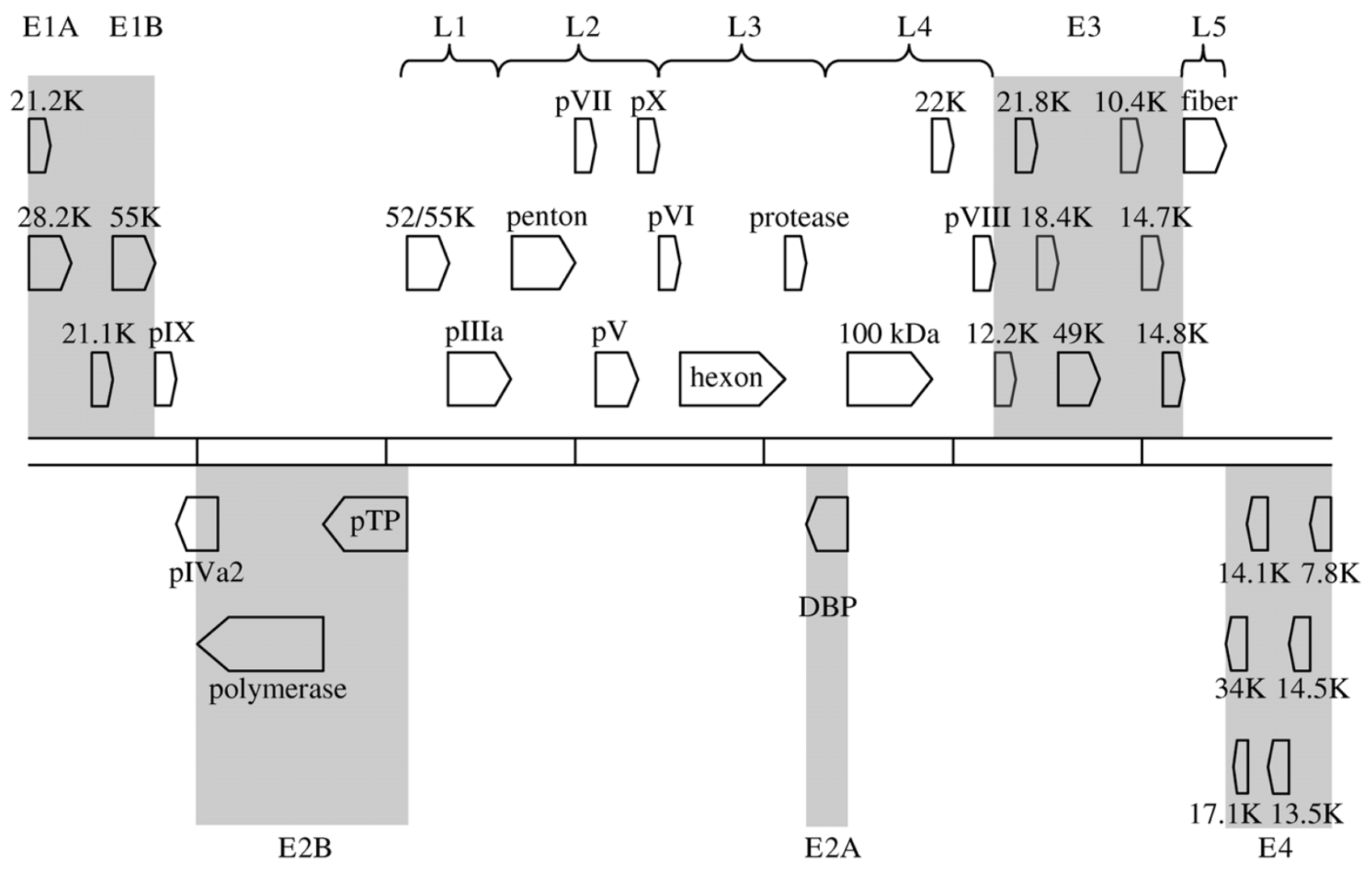

Figure I

Transcriptional map and genome organization of HAdV-37. The two horizontal lines define the length of the HAdV37 genome with each vertical line within them representing 5000 bps. The block arrows represent the predicted protein coding regions. The early transcription units (EI-E4) are highlighted. The late transcription units (LI-L5) are designated by parentheses.

E1B proteins potentiate viral replication by blocking apoptosis. E1B 19K blocks the mitochondrial apoptosis pathway by inactivating BAK and BAX [19]. E1B 55K inhibits the ability of p53, the host tumor suppressor protein, to initiate cell cycle arrest $[20,21]$. The putative TATA box for the E1B messages was predicted at nucleotide 1525. Two predicted proteins of molecular weights of 21.1 and $55.2 \mathrm{kDa}$ were identified within E1B which correspond to 19 - and $55-\mathrm{kDa}$ proteins, respectively, as reported for HAdV-9. Amino acid sequence analysis revealed that the predicted $21.1 \mathrm{kDa}$ protein was $99 \%$ identical and $100 \%$ similar to the $19 \mathrm{kDa}$ homologue found in the HAdV-9 genome (Table 2). The polyadenylation signal for these transcripts was predicted at nucleotide 3863.

The E2 region of the genome consists of two transcription units, E2A and E2B, which encode three proteins that are required for viral replication [7]. These three proteins are known as the DNA binding protein (DBP), terminal protein precursor (pTP), and DNA polymerase. The E2A 54.9 $\mathrm{kDa}$ DNA binding protein was identified on the comple- mentary strand between nucleotides 21305 and 22777 . Also on the complementary strand, but located within the E2B region, we identified the pTP and DNA polymerase. The polyadenylation signal for these transcripts was not identified.

The HAdV E3 region encodes proteins that modulate the host immune response to infection but are not required for viral growth in vitro $[22,23]$. HAdVs within species D have previously been suggested to encode eight ORFs within the E3 region [24,25]. Seven classical and one hypothetical E3 ORFs were identified in our annotation of HAdV-37. The predicted molecular weights for these are $12.2,21.8,18.6,48.9,31.6,10.47,14.7$, and $14.8 \mathrm{kDa}$. The TATA box was predicted at nucleotide 25879 with a TATAAA motif. One polyadenylation signal for this transcription unit was identified at nucleotide 30837.

Open reading frames located in the $\mathrm{E} 4$ transcription unit produce proteins that have a wide variety of functions [26]. For example, E4 ORF 3 and E4 ORF 6 enhance the stability of late viral mRNAs and increase their export 

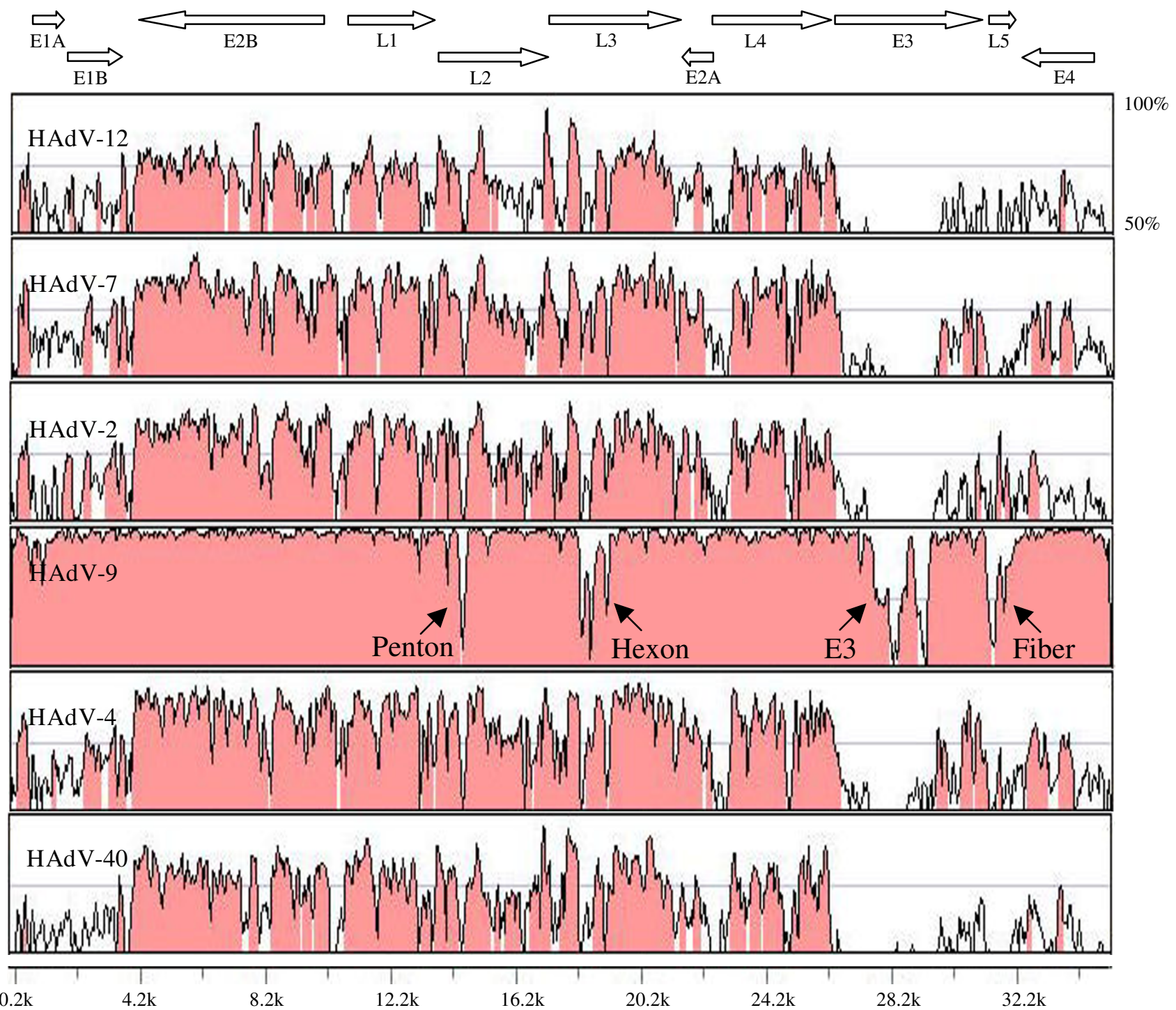

Figure 2

Global pairwise sequence comparison of HAdV-37 with select serotypes from each of the $6 \mathrm{HAdV}$ species (from top to bottom: species $A$ to $F$ ) using the online sequence alignment program, mVISTA LAGAN. Percent sequence conservation is reflected in the height of each data point along the $y$ axis. The penton, hexon, E3, and fiber regions of HAdV-37 diverged from HAdV-9, another species D virus.

from the nucleus thereby increasing viral mRNA accumulation in the cytoplasm [26]. E4 ORF 6 also binds to p53 and can block apoptosis $[27,28]$. We found 6 predicted ORFs in HAdV-37 located on the complementary strand. Surprisingly, the E4 ORF 1 from the HAdV-37 genome was predicted at 65 amino acids in length corresponding to a molecular weight of $7.4 \mathrm{kDa}$. In contrast, the HAdV-9 homologue of $\mathrm{E} 4 \mathrm{ORF} 1$ is 125 amino acids in length, and contains three regions essential for tumor transformation (region I, residues 34 to 41; region II, residues 89 to 91; region III 122 to 125). The E4 ORF 1 of HAdV-9 has struc- tural similarity to other viral dUTPase enzymes [29,30]. ClustalW analysis of the HAdV-37 E4 ORF 1 compared to the HAdV-9 homologue revealed a $100 \%$ similarity from residues 61-125, including regions II and III and a truncated dUTPase domain. Further work will be needed to evaluate the significance of this truncation. The TATA box for this region was identified at nucleotide 34665 and the polyadenylation signal at nucleotide 32184 . 
Table I: Genome Organization of HAdV-37

\begin{tabular}{|c|c|c|c|c|c|c|}
\hline Region & Gene Product & TATA box & ATG & Stop & Protein Length $(\mathrm{aa})$ & Poly(A) signal \\
\hline \multirow[t]{2}{*}{ EIA } & $21.2 \mathrm{kDa}$ & 477 & $569-932^{*}$ & $1214-1425 *$ & 191 & $|45|$ \\
\hline & $28.2 \mathrm{kDa}$ & 477 & $569-1118^{*}$ & $1214-1425^{*}$ & 253 & $|45|$ \\
\hline \multirow[t]{2}{*}{ EIB } & $21.1 \mathrm{kDa}$ & 1525 & 1577 & 2125 & 182 & 3863 \\
\hline & $55.2 \mathrm{kDa}$ & 1525 & 1882 & 3369 & 495 & 3863 \\
\hline \multirow[t]{5}{*}{ Intermediate } & IX & ND & 3454 & 3858 & 134 & 3863 \\
\hline & hypothetical 20.5 kDa & ND & $5765 c$ & $5199 c$ & 188 & ND \\
\hline & $\mathrm{IV} a 2$ & ND & $5514-5526 *_{c}$ & $3902-5235 *_{c}$ & 448 & ND \\
\hline & hypothetical 34.76 kDa & ND & 6514 & 7503 & 329 & ND \\
\hline & hypothetical 16.57 kDa & ND & 7786 & 8253 & 155 & ND \\
\hline E2A & DNA binding protein & ND & $22777 c$ & $21305 c$ & 490 & ND \\
\hline \multirow[t]{4}{*}{ E2B } & PTP & ND & $13495-13503 * c$ & $8325-10223 *_{c}$ & 635 & ND \\
\hline & hypothetical 19.29 kDa & ND & $8030 c$ & $8563 \mathrm{c}$ & 177 & ND \\
\hline & DNA Polymerase & ND & $8280 c$ & $5005 c$ & 1091 & ND \\
\hline & hypothetical II.4 kDa & ND & 9648 & 9983 & 111 & ND \\
\hline \multirow[t]{3}{*}{ LI } & $52 / 55 \mathrm{~K}$ & 5827 & 10638 & 11762 & 374 & 13484 \\
\hline & hypothetical 8.58 kDa & ND & $|I 78| c$ & $12035 c$ & 84 & ND \\
\hline & pllla & 5827 & 11785 & 13476 & 563 & 13484 \\
\hline \multirow[t]{4}{*}{ L2 } & Penton & 5827 & 13530 & 15089 & 519 & 16982 \\
\hline & pVII & 5827 & 15093 & 15683 & 196 & 16982 \\
\hline & $\mathrm{V}$ & 5827 & 15716 & 16720 & 334 & 16982 \\
\hline & $\mathrm{pX}$ & 5827 & 16750 & 16974 & 74 & 16982 \\
\hline \multirow[t]{3}{*}{ L3 } & $\mathrm{pVI}$ & 5827 & 17030 & 17734 & 234 & 21256 \\
\hline & Hexon & 5827 & 17775 & 20642 & 949 & 21256 \\
\hline & Protease & 5827 & 20627 & 21250 & 207 & 21256 \\
\hline \multirow[t]{4}{*}{ L4 } & I0OK & 5827 & 22794 & 24992 & 732 & 26507 \\
\hline & hypothetical 9.29 kDa & ND & $24181 \mathrm{c}$ & $23936 c$ & 81 & ND \\
\hline & $22 \mathrm{~K}$ & 5827 & 24775 & 25188 & 137 & 26507 \\
\hline & pVIII & 5827 & 25514 & 26197 & 227 & 26507 \\
\hline \multirow[t]{9}{*}{ E3 } & $12.2 \mathrm{kDa}$ & 25879 & 26198 & 26518 & 106 & 30837 \\
\hline & $21.8 \mathrm{kDa}$ & 25879 & 26472 & 27062 & 196 & 30837 \\
\hline & 18.6 kDa & 25879 & 27029 & 27529 & 166 & 30837 \\
\hline & $48.9 \mathrm{kDa}$ & 25879 & 27554 & 28840 & 428 & 30837 \\
\hline & hypothetical 31.6 kDa & 25879 & 28867 & 29709 & 280 & 30837 \\
\hline & 10.4 kDa & 25879 & 29716 & 29991 & 91 & 30837 \\
\hline & $14.72 \mathrm{kDa}$ & 25879 & 29994 & 30386 & 130 & 30837 \\
\hline & $14.84 \mathrm{kDa}$ & 25879 & 30379 & 30771 & 130 & 30837 \\
\hline & U-exon & ND & $31022 c$ & $30873 c$ & 49 & ND \\
\hline L5 & Fiber & 5827 & 31038 & 32135 & 365 & 32143 \\
\hline \multirow[t]{6}{*}{ E4 } & $7.8 \mathrm{kDa}$ & 34665 & $34564 c$ & $34367 c$ & 65 & 32184 \\
\hline & $14.5 \mathrm{kDa}$ & 34665 & $34326 c$ & $33934 c$ & 130 & 32184 \\
\hline & I3.56 kDa & 34665 & $33937 c$ & $33584 c$ & 117 & 32184 \\
\hline & $14.16 \mathrm{kDa}$ & 34665 & $33581 \mathrm{c}$ & $33219 c$ & 120 & 32184 \\
\hline & $17.13 \mathrm{kDa}$ & 34665 & $33122 c$ & $32667 c$ & 151 & 32184 \\
\hline & 34.I kDa & 34665 & $33289 c$ & $32411 \mathrm{c}$ & 292 & 32184 \\
\hline
\end{tabular}

Note: c, complementary strand

*, predicted splice sites

\section{Intermediate genes}

The intermediate genes of HAdV are IVa2 and IX. The IVa2 protein interacts with L1 52/55K during viral DNA packaging, and assists in the activation of the major late promoter (MLP) [14,31-33]. The HAdV-37 IVa2 gene, found on the complementary strand, was predicted using the splice site finder [34], with a 448 amino acid protein and 99\% amino acid homology to HAdV-9 IVa2. The IX protein is a minor capsid protein and also assists in the activation of the major late promoter $[35,36]$. A coding sequence for a $13.7 \mathrm{kDa}$ protein corresponding to IX was found at nucleotides 3454-3858.

\section{Late genes}

The late transcription units of HAdVs are transcribed from the MLP, which consists of an inverted CAAT box (57775780 bp) and TATA box (5827-5832 bp). The late mRNAs have been grouped into five families (L1 to L5), based on the location of the polyadenylation signal. Proteins expressed by these five families are involved in cap- 
Table 2: Percent identities/similarities of select HAdV-37 proteins and their homologs

\begin{tabular}{|c|c|c|c|c|c|c|c|}
\hline Types/Species & EIA $21.2-k D a$ & EIB 2I.I-kDa & IX & DNA binding protein & DNA polymerase & LI 52/55K & Penton \\
\hline HAdV-I2/A & $4 I / 65$ & $46 / 77$ & $56 / 79$ & $52 / 78$ & $78 / 91$ & $78 / 92$ & $74 / 86$ \\
\hline HAdV-7/B & $4 I / 6 I$ & $53 / 81$ & $63 / 85$ & $61 / 82$ & $82 / 93$ & $79 / 88$ & $75 / 89$ \\
\hline HAdV-2/C & $35 / 46$ & $50 / 78$ & $52 / 83$ & $57 / 76$ & $83 / 93$ & $75 / 88$ & $68 / 82$ \\
\hline HAdV-9/D & $89 / 96$ & $97 / 100$ & $99 / 100$ & $98 / 99$ & $99 / 99$ & $99 / 100$ & $90 / 95$ \\
\hline HAdV-4/E & $42 / 63$ & $50 / 79$ & $62 / 86$ & $60 / 79$ & $83 / 93$ & $77 / 87$ & $77 / 88$ \\
\hline HAdV-40/F & $35 / 56$ & $43 / 72$ & $55 / 81$ & $51 / 75$ & $74 / 90$ & $81 / 91$ & $73 / 86$ \\
\hline Types/Species & Hexon & L3 Protease & L4 I00K & L4 pVIII & E3 $14.7-\mathrm{kDa}$ & Fiber & E4 $14.5-\mathrm{kDa}$ \\
\hline HAdV-I2/A & $80 / 90$ & $79 / 93$ & $66 / 83$ & $75 / 88$ & $35 / 63$ & $32 / 66$ & $35 / 66$ \\
\hline HAdV-7/B & $85 / 93$ & $84 / 93$ & $69 / 84$ & $82 / 93$ & $47 / 68$ & $32 / 57$ & $42 / 78$ \\
\hline HAdV-2/C & $78 / 90$ & $77 / 94$ & $69 / 83$ & $78 / 91$ & $35 / 64$ & $36 / 65$ & $32 / 71$ \\
\hline HAdV-9/D & $90 / 95$ & $100 / 100$ & $98 / 99$ & $99 / 100$ & $98 / 98$ & $76 / 89$ & $100 / 100$ \\
\hline HAdV-4/E & $85 / 93$ & $82 / 93$ & $71 / 87$ & $84 / 94$ & $38 / 62$ & $37 / 62$ & $40 / 75$ \\
\hline $\mathrm{HAdV}-40 / \mathrm{F}$ & $80 / 92$ & $78 / 94$ & $66 / 84$ & $80 / 90$ & $30 / 53$ & $31 / 59$ & $37 / 66$ \\
\hline
\end{tabular}

Percent identities and similarities were calculated using FASTA3 (EMBL-EBI).

sid production for mature virions [7]. The $\mathrm{L} 1$ transcription unit encodes two proteins, 52/55K and IIIa. The 52/55K protein is involved in scaffolding of the capsid and therefore facilitates virus assembly [37]. The 52/55K protein also interacts with the intermediate gene product IVa2 to facilitate DNA packaging $[31,33,38]$. Polypeptide IIIa is a structural protein that has been located on the inner capsid surface below the penton base [39]. The 52/55K and polypeptide IIIa in HAdV-37 were predicted to have molecular weights of $42.2 \mathrm{kDa}$ and $26.6 \mathrm{kDa}$, respectively. The predicted polyadenylation signal for the L1 region was found at nucleotide 13484 .

The proteins encoded on the $\mathrm{L} 2$ transcription unit also are involved in capsid formation [7]. The penton base (protein III) is found at each of the 12 vertices of the virion [7]. The penton base contains an Arg-Gly-Asp (RGD) sequence which interacts with host integrins to induce internalization of the virus [40]. The HAdV-37 penton base is located at nucleotides 13530-15089. The length of the protein was predicted to be 519 amino acids with an estimated molecular weight of $58.4 \mathrm{kDa}$. The RGD sequence was located at amino acid position 309-311. The predicted protein was $100 \%$ identical to the previously published penton base protein identified for HAdV37 [41]. The HAdV-37 penton base homologue is $90 \%$ identical and 95\% similar to the predicted HAdV-9 penton base protein (Table 2). The V, VII, and X proteins constitute the HAdV core proteins and facilitate packaging of viral DNA within the capsid [42]. The HAdV-37 pVII protein-coding sequence was identified at nucleotides 15093-15683, and the protein was predicted to have a molecular weight of $21.7 \mathrm{kDa}$. Proteins with an amino acid length of 334 and 74 were predicted in the HAdV-37 genome for proteins $\mathrm{V}$ and $\mathrm{X}$ at nucleotides 15716-16720 and 16750-16974, respectively. The $\mathrm{L} 2$ transcripts share a putative polyadenylation signal at nucleotide 16982 .

Three open reading frames corresponding to pVI, hexon, and protease proteins, with respective molecular weights of $25.5,106.8$, and $23.4 \mathrm{kDa}$, were identified within the L3 transcription unit. The pVI protein contains two nuclear export signals and two nuclear localization signals, and plays a role in transporting the hexon protein to the nucleus for viral assembly [43]. The $\mathrm{C}$ terminus of this protein has also been implicated in regulation of the viral protease [44]. The HAdV-37 pVI protein was located at nucleotides 17030-17734. This predicted 234 amino acid protein was $100 \%$ identical to its HAdV-17 homologue. The hexon protein, the most abundant virion component, constitutes 240 of the 252 subunits of the protein shell of the virus [7]. The HAdV-37 hexon protein is 949 amino acids in length and was nearly identical to that predicted by Ebner et al. [45]. Our sequence data suggests an additional 10 amino acids on the N-terminus of the protein, similar to the predicted hexon gene for HAdV-46 and HAdV-9, both within species D. The final protein encoded in the L3 transcription unit is the $23 \mathrm{kDa}$ viral protease protein. The HAdV-37 homologue was predicted to be 207 amino acids in length. This protein cleaves other viral proteins allowing for assembly and viral maturation [46], and the transcript shares a predicted polyadenylation signal at nucleotide 21256 with other L3 transcripts.

Three L4 proteins, $100 \mathrm{kDa}$, pVIII, and $22 \mathrm{kDa}$, were predicted from our annotation. The $100 \mathrm{kDa}$ protein is a nonstructural protein that assists in the translation of late viral mRNAs, and inhibits translation of cellular mRNAs $[47,48]$. More recently this protein has been implicated as a scaffold for trimerization of the hexon [49]. The pre- 
dicted $100 \mathrm{kDa}$ protein for HAdV-37 genome was 732 amino acids in length and had a molecular weight of 82.3 $\mathrm{kDa}$. This protein is $98 \%$ identical to the published HAdV$46100 \mathrm{kDa}$ protein. Protein VIII is a minor capsid protein that plays a role in the stability of the virion capsid [50]. The pVIII protein of HAdV-37 is $24.6 \mathrm{kDa}$ in molecular weight and has a 99\% identity to the published HAdV-46 pVIII protein. The $22 \mathrm{kDa}$ protein is involved in the packaging of HAdV DNA [51]. A $22 \mathrm{kDa}$ homologue was identified in HAdV-37 with a predicted protein of 137 amino acids and molecular weight of $15.8 \mathrm{kDa}$. Its highest percent identity was to the HAdV-9 $22 \mathrm{kDa}$ protein (99\%). The predicted polyadenylation signal for the L4 transcription unit is at nucleotide 26507.

The L5 region of the HAdV genome consists entirely of the fiber protein gene. Fiber protein trimerizes to produce the functional unit which projects from the 12 penton vertices of the virus capsid. The fiber protein's carboxyl (C)-terminal globular domain, known as the fiber knob, acts as the primary ligand for host cell receptor binding. The HAdV37 fiber genome sequence was previously reported, and our predicted protein of 365 amino acids was identical [52]. The HAdV-37 fiber was only $76 \%$ identical and $89 \%$ similar to its homologue in the HAdV-9 genome (Table 2 ). The polyadenylation signal for this transcript was predicted at nucleotide 32143. Nucelotide sequence encoding a potential heparan binding site, previously reported in the fiber shaft of HAdV-5, was not present in the HAdV37 fiber gene [53-55].

\section{Virus-associated RNA}

Most HAdVs contain two virus-associated (VA) RNA genes, VA RNAI and VA RNAII. VA RNAI acts against cellular antiviral defense by blocking the activation of the protein kinase PKR, which when activated turns off protein synthesis in infected cells [56]. VA RNAII binds to RNA helicase A and NF90, the latter a component of the nuclear factor of activated T cells (NFAT) [57]. These VA RNAs also have been recently shown to suppress RNA interference [58]. The VA RNA genes for HAdV-37 were previously identified [59]. Our sequence for VA RNAI is located at nucleotides $10253-10410$ and is $99 \%$ identical to the previously reported sequence, differing by only one base pair. VA RNAII is located at nucleotides 1047110620 and was $100 \%$ identical to that previously reported.

\section{Protein and phylogenetic analysis}

The annotation of the HAdV-37 genome allows for its comparison with other HAdV serotypes within species D as well as serotypes from other species. Percent identity and similarity of predicted proteins from each of the major transcription units were identified for representative serotypes using Fasta3 [60], and are shown in Table 2.
In this analysis, highest identities outside of species $\mathrm{D}$ were seen with species B (HAdV-7) and species E (HAdV4) viruses. Projected protein sequences were then subjected to phylogenetic analysis using Molecular Evolutionary Genetics Analysis (MEGA) 3.1. Bootstrap confirmed neighbor joining trees also suggested that outside of HAdV species $D$, the serotypes phylogenetically closest to HAdV-37 were within HAdV species B and E (Figure 3). We further selected specific proteins for analysis by virtual 2D gel (JVirGel 2.2.3b) [61,62], based on ClustalW alignments of predicted protein amino acid sequences comparing serotypes from different HAdV species. The accuracy of these virtual 2D gels with regards to pI has been judged to be within \pm 1 pI unit of the true migration of the physical protein, even when subsequent post-translational modifications are taken into account $[61,63,64]$.

Migration patterns for select protein homologues in the virtual 2D gel showed projected differences in size and/or pI (See Additional file 1: Supplemental figure 4). The HAdV-37 DNA binding protein migrated to a predicted molecular weight of $54.9 \mathrm{kDa}$ and a pI of 8.52 (Table 3 and Additional file 1). The range of pI for the DNA binding protein among all serotypes tested was from 6.30 to 8.57. The DNA polymerase homologues also revealed substantial differences in predicted size among the selected serotypes, and a range in $\mathrm{pI}$ from 6.19 to 8.18 (Table 3). HAdV-37 and HAdV-9 polymerase both migrated to a predicted molecular weight of $125 \mathrm{kDa}$ with pI's of 6.28 and 6.19, respectively. The HAdV-40 homologue had a predicted $\mathrm{pI}$ of 8.14 . The predicted molecular weights of the penton and hexon proteins differed between serotypes by less than $10 \mathrm{kDa}$, with a pI range that was probably within the range of accuracy of the software (Table 3 and additional file 1). The L3 protease homologues migrated to almost identical areas on the virtual gel (Additional file 1), consistent with very high percent similarity between HAdV-37 protease and the other homologues (93 to $100 \%$, Table 2). In contrast, despite high percent similarity in the pVIII protein between HAdV-37 and HAdV-4 (94\%), the predicted HAdV-37 pVIII migrated to a pI of 8.80 , while the HAdV-4 pVIII migrated to a pI of 6.22 (Table 2 and Additional file 1). Further review of the ClustalW alignment for these 2 homologues revealed that despite their high similarity, there were 3 specific amino acid differences in HAdV-37 that when changed to match the residues in HAdV-4, resulted in a pI for HAdV-37 of 5.78 (G46D, Q57E, Q172E, data not shown).

\section{Hypothetical proteins}

During annotation of HAdV-37, we located 8 hypothetical ORFs similar to ORFs predicted from sequences previously archived in GenBank for other HAdVs (Table 4), 
DNA Binding Protein

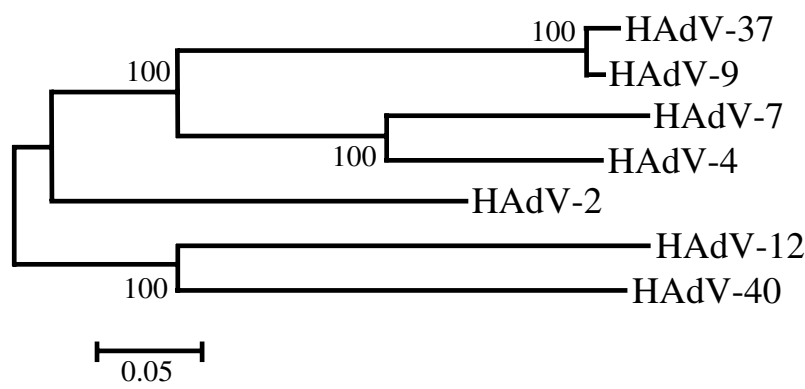

Penton

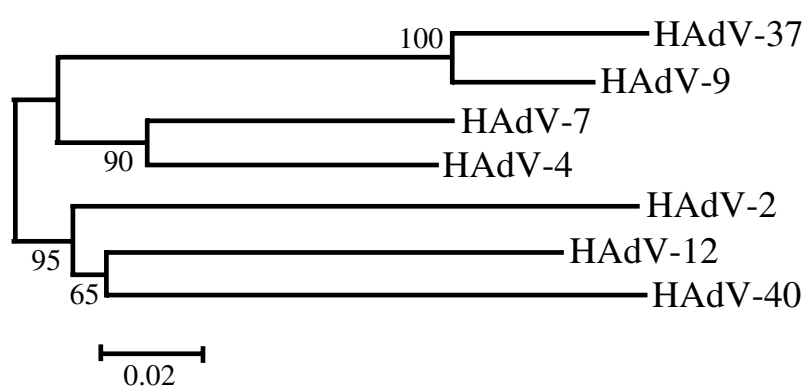

Protease

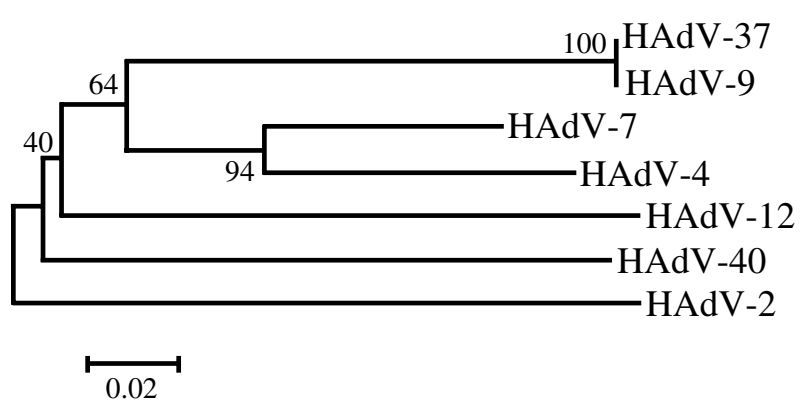

Polymerase

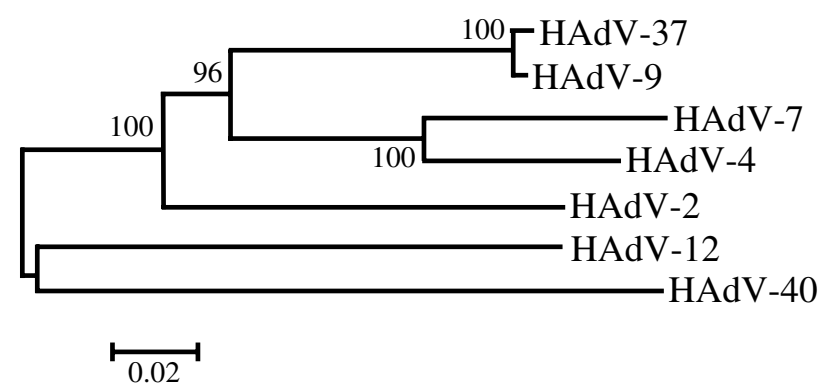

Hexon

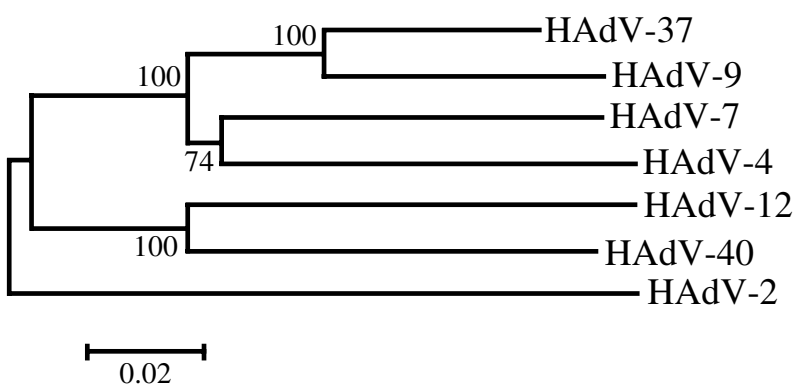

pVIII

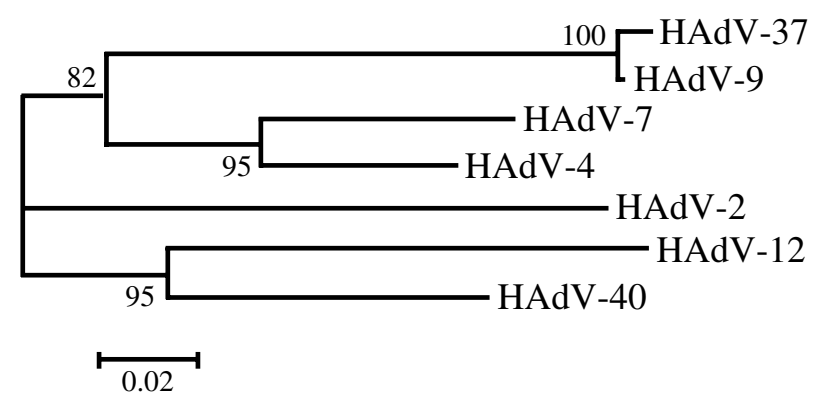

Figure 3

Phylogenetic analysis of select HAdV proteins. Bootstrap confirmed neighbor joining trees designed from MEGA 3.I demonstrate phylogenetic relationships between select proteins of HAdV-37 and representative homologues from each of the 6 HAdV species. The Gonnet protein weight matrix in ClustalX alignment was used, along with complete deletion options. Bootstrap confidence levels (500 replicates) are shown as percentages on the relevant branches.

with a blast value for each of less than $e^{-5}$. GeneMark identified one of these putative proteins (HAdV-7 $13.6 \mathrm{kDa}$ agnoprotein), and JCVI's annotation engine identified another (E3B $31.6 \mathrm{kDa}$ ), while the rest were identified by NCBI's ORF finder. Four of the 8 proteins were located on the complementary strand and 5 were clustered in the area between the intermediate and late ORFs.

\section{Discussion}

We have determined the complete 35,213 base pair genome of HAdV-37 and identified 35 putative adenoviral genes along with 8 hypothetical ORFs conserved with at least one other HAdV for each ORF. Comparison of the HAdV-37 genome to that of HAdV-9, another species D virus, identified areas of substantial divergence in the penton, hexon, E3, and fiber regions. Disparities between these two HAdV species D viruses in genes encoding struc- 
Table 3: Molecular weight/pl of select HAdV-37 proteins and their homologs in other HAdV species

\begin{tabular}{|c|c|c|c|c|c|c|}
\hline Types/Species & DNA binding protein & DNA polymerase & Penton Base & Hexon & Protease & $\mathrm{pVIII}$ \\
\hline HAdV-I2/A & $55.0 / 7.19$ & $135.0 / 7.50$ & $56.4 / 6.29$ & $103.0 / 5.56$ & $23.5 / 8.50$ & $25.3 / 9.42$ \\
\hline HAdV-7/B & $58.2 / 8.57$ & $136.0 / 6.87$ & $61.9 / 5.37$ & $105.7 / 5.13$ & $23.7 / 8.69$ & $24.9 / 6.12$ \\
\hline $\mathrm{HAdV}-2 / \mathrm{C}$ & $59.1 / 8.06$ & $120.4 / 6.22$ & $63.2 / 5.10$ & $|09.2 / 4.8|$ & $23.1 / 8.68$ & $24.7 / 9.52$ \\
\hline HAdV-9/D & $54.8 / 8.52$ & $125.4 / 6.19$ & $58.6 / 5.21$ & $106.3 / 5.00$ & $23.6 / 8.61$ & $24.6 / 8.15$ \\
\hline HAdV-37/D & $54.9 / 8.52$ & $125.4 / 6.28$ & $58.4 / 5.47$ & $106.8 / 5.6$ & $23.4 / 8.61$ & $24.6 / 8.80$ \\
\hline HAdV-4/E & $57.2 / 8.11$ & $116.7 / 6.33$ & $60.0 / 4.92$ & $105.4 / 5.25$ & $23.4 / 8.13$ & $24.7 / 6.22$ \\
\hline HAdV-40/F & $53.3 / 6.30$ & $135.1 / 8.14$ & $56.9 / 5.84$ & $104.0 / 5.74$ & $23.3 / 8.68$ & $25.3 / 8.47$ \\
\hline
\end{tabular}

Values in bold represents pl's +/- > I pH unit from predicted HAdV-37 proteins

Molecular weight and $\mathrm{pl}$ were calculated using JVirGel 2.2.3b

tural and host receptor-binding proteins were somewhat expected and also consistent with known differences in host tissue tropism, for example the propensity of HAdV37 to cause corneal infection, as compared to the association of HAdV-9 with urethritis and follicular conjunctivitis $[7,65]$. Differences between HAdV-9 and 37 in the E3 region, known to be important to immune evasion and regulation by the virus, but not essential to viral replication in vitro, suggest as yet undiscovered functions for this region [22,23]. Divergence in the $\mathrm{E} 3$ region, possibly relevant to cellular and tissue specificity during infection, might be due to positive selection. Sequencing of other HAdVs within species D would provide further insight into this area of the HAdV genome.

By phylogenetic analyses and paired comparisons of predicted proteins, HAdV-37 and HAdV-9 of species D appeared most closely related to HAdV-7 of species B and HAdV-4 of species E. Subsequent virtual 2D gel analyses suggested that for a few proteins, a relatively few amino acid substitutions between otherwise similar proteins conferred significant effects on protein charge. If our analyses prove correct, such differences suggest that the function of such proteins in HAdV species D could be quite different than previously described for serotypes of other HAdV species. We acknowledge that our predictions represent a first approximation of protein characteristics, and could be subject to over-interpretation for at least two reasons. First, our comparisons to other viruses are only as reliable as the quality of GenBank viral sequence and annotation. Secondly, post-translational modifications may alter both charge and molecular weight of any given protein. Actual 2D gel analysis will be necessary to confirm such predicted differences.

There is growing concern over the accuracy of in silico ORF prediction in AdVs due to splice variants, as well as inconsistencies in banked annotations [66]. To address such concerns, we compared HAdV-37 annotation using three different methods: NCBI ORF finder, JCVI's annotation engine, and GeneMark Heuristic model. We narrowed our annotation to 35 ORFs by comparison with previously determined adenoviral annotations, but we consider our annotation provisional. We identified 8 hypothetical ORFs similar to those previously identified in other HAdV species. The very suggestion of hypothetical proteins implies that our understanding of the HAdV is far from complete. Transcriptome analysis using viral microarrays may help to clarify the best annotation [67]. We suggest that the true transcriptome and proteome of HAdV-37 remain to be determined.

Future sequencing of HAdVs may permit new insights into viral origin, evolution, and pathogenesis. Recently, HAdV22 was isolated for the first time from an outbreak of epidemic keratoconjunctivitis. The HAdV-22 isolate was shown to contain both HAdV-8 fiber gene and HAdV-37 penton base gene [68]. These recombination events

Table 4: Conserved hypothetical HAdV-37 Proteins

\begin{tabular}{|c|c|c|c|}
\hline Predicted Size & Blast Result & E-Value & Accession Number \\
\hline $20.5 \mathrm{kDa}$ & HAdV-5 Hypothetical 12 kDa & $5.0 \mathrm{E}-20$ & $\underline{\text { AAW } 65500}$ \\
\hline $34.76 \mathrm{kDa}$ & HAdV-7 Hypothetical 10.4 kDa & $7.0 \mathrm{E}-23$ & AAW33389 \\
\hline 16.57 kDa & HAdV-7 I3.6 Agnoprotein & $2.0 \mathrm{E}-39$ & AAT97609 \\
\hline $19.29 \mathrm{kDa}$ & HAdV-I6 Hypothetical 12.6 kDa & $9.0 \mathrm{E}-12$ & AAW33435 \\
\hline $1 \mathrm{l} .4 \mathrm{kDa}$ & HAdV-3 Hypothetical 9.7 kDa & $1.0 \mathrm{E}-9$ & AAW33161 \\
\hline $8.58 \mathrm{kDa}$ & HAdV-7 Hypothetical I I.3 kDa & $7.0 \mathrm{E}-14$ & AAT97539 \\
\hline $9.29 \mathrm{kDa}$ & HAdV-7 Hypothetical protein & $2.0 \mathrm{E}-20$ & AAT97549 \\
\hline $31.6 \mathrm{kDa}$ & HAdV-9 E3 orf3 33.I kDa & $3.0 \mathrm{E}-65$ & $\overline{\text { CAI0598I }}$ \\
\hline
\end{tabular}


apparently conferred corneal tropism to HAdV-22, a virus not normally known to infect the cornea. As more HAdV species $D$ viruses are sequenced, new insights into tropism and pathogenesis are likely to emerge.

\section{Conclusion}

In summary, the complete genome sequence of HAdV-37 was determined and annotated. The organization of the HAdV-37 genome is similar to other human species D adenoviruses except in the penton, hexon, E3, and fiber regions. Phylogenetic analysis of HAdV-37 proteins revealed close relation to species $B$ and $E$ human adenoviruses, while virtual 2D gel analysis identified differences in proteins thought to function similarly. The availability of the HAdV-37 complete genome sequence will facilitate future studies into the pathogenicity of this important human pathogen.

\section{Methods}

\section{Cells, virus stock, DNA purification}

HAdV-37 strain GW was obtained from the American Type Culture Collection (ATCC). Virus stocks were grown in A-549 cells (CCL-185), a human alveolar epithelial cell line that was previously shown to support HAdV-1 virion production [69]. Virus was purified by $\mathrm{CsCl}$ gradient and subsequent dialysis, and stored at $-80^{\circ} \mathrm{C}$. DNA extraction was accomplished by the addition of proteinase $\mathrm{K}$, phenol:chloroform extraction, and finally ethanol precipitation.

\section{Sequencing}

Standard PCR methodology was used to amplify regions of the genome to be sequenced. HAdV type 17 was used as a reference strain for the design of initial PCR primers. To close gaps in the sequence and improve overall sequence quality, Primer 3 [70] and CONSED [71] software were used to design primers from newly acquired sequence. Shrimp alkaline phosphatase and exonuclease I treatment were used to dephosphorylate and degrade residual PCR primers present together with the PCR products. Sequencing was performed using the ABI BigDye Terminator v3.1 cycle sequencing kit (Applied Biosystems, Foster City, $\mathrm{CA}$ ). The sequencing reaction mixture was purified using Sephadex G-50 (Sigma Aldrich, St. Louis, MO), and the reaction products analyzed on $\mathrm{ABI} 3700$ or $\mathrm{ABI} 3730 \mathrm{XL}$ capillary electrophoresis DNA sequencers (Applied Biosystems). To sequence the viral inverted terminal repeat (ITR) ends, primers were designed from newly determined adjacent sequence, and direct sequencing was performed using whole genome DNA as the template [69].

\section{Sequence analysis and genome annotation}

Sequence data was filtered using LUCY (JCVI, Rockville, MD), and data assembly performed with Phred/Phrap, using default assembly parameters [71-73]. Genome assembly contained 664 high quality reads with an average length of $834 \mathrm{bps}$. The fold coverage for both strands of the genome was 15 . The Phrap average quality score was 89.0. Genome annotation was performed using JCVI's automated annotation system [74], and the data was stored in a MySQL database. Manatee [75] was used to manually review the data from the annotation engine. Additionally, we used GeneMark Heuristic Models gene prediction [76], and NCBI's ORF Finder [77] to examine the sequence. Open reading frames were searched against available databases in GenBank, PIR, SWISS-PROT, and JCVI's CMR database. Splice sites were predicted using a splice site finder program [34]. An online sequence alignment program, mVISTA LAGAN [78] was used for global pair-wise sequence alignment [15]. CpG analysis was performed with FUZZNUC [12].

\section{Nucleotide sequence accession numbers}

The nucleotide sequence for the following HAdVs can be found in GenBank: HAdV-2 [AC_000007], HAdV-4 [AY487947], HAdV-7 [ [AC 000018], HAdV-9 [ㅍ854486], HAdV-12 [ $\underline{\text { AC 000005] }}$, HAdV-17 [AC 000006], HAdV40 [L19443]. Previously sequenced HAdV-37 penton base protein, hexon protein, fiber protein, and VA RNA gene accession numbers are AAG00906, ABA00016, $\underline{\mathrm{AAB} 71734}$, and $\underline{\mathrm{U} 10679}$, respectively. The GenBank accession number for HAdV-37 is DQ900900.

\section{In silico protein analysis}

Percent identities and similarities between proteins of HAdV-37 and other HAdVs were determined using Fasta3 $[60,79]$ and Blastp software [80]. Proteins from the GenBank database were analyzed by an in silico 2D gel program JVirGel 2.2.3b [61]. Phylogenetic analysis was performed with Molecular Evolutionary Genetics Analysis (MEGA) 3.1 [81]. Bootstrap confirmed neighbor joining phylogenetic trees were designed with MEGA 3.1 with 500 replicates.

\section{Authors' contributions}

CMR designed primers, annotated the virus, performed the bioinformatics analysis, and drafted the manuscript. FS performed the PCR, and assisted with compilation of the sequence. AFG and DWD participated in primer design, sequence compilation and analysis, and manuscript writing. JC conceived the project design, and participated in the data analysis writing of the manuscript. All authors read and approved the final manuscript. 


\section{Additional material}

\section{Additional file 1}

2D Gel Analysis. Virtual 2D gel analysis. Protein migration patterns for select HAdV proteins by virtual $2 \mathrm{D}$ gel. Each spot represents a given serotype's homologue based on its predicted amino acid sequence. A. DNA binding protein, B. Viral polymerase, C. Penton base, D. Hexon, E. Protease, and F. pVIII. One protein from HAdV-37 and a homologue from a representative serotype of all $6 \mathrm{HAdV}$ species are represented in each gel. Click here for file

[http://www.biomedcentral.com/content/supplementary/14712164-9-213-S1.ppt]

\section{Acknowledgements}

We thank Nicole Benton for her technical assistance in sequencing as well as Jeremy Zaitshik for his bioinformatics assistance. Research support was provided through NIH grants EYOI3 I24, EYOI5222, EYOI 2190, P2O RR017703, P20 RR015564, T32 AI007633 and a Research to Prevent Blindness Physician-Scientist Merit Award (to JC).

\section{References}

I. Benko M B. Harrach, and W. C. Russell: Family Adenoviridae. In Virus Taxonomy: Seventh Report of the International Committee on Taxonomy of Viruses Edited by: M. H. V. van Regenmortel CMFDHLBEBCMKESMLJMMAMDJMGCRPRBW. San Diego , Academic Press; 2000:227-238.

2. Hilleman MR, Werner JH: Recovery of new agent from patients with acute respiratory illness. Proceedings of the Society for Experimental Biology and Medicine Society for Experimental Biology and Medicine (New York, NY 1954, 85(I): I83-188.

3. Rowe WP, Huebner RJ, Gilmore LK, Parrott RH, Ward TG: Isolation of a cytopathogenic agent from human adenoids undergoing spontaneous degeneration in tissue culture. Proceedings of the Society for Experimental Biology and Medicine Society for Experimental Biology and Medicine (New York, NY 1953, 84(3):570-573.

4. Dingle JH, Langmuir AD: Epidemiology of acute, respiratory disease in military recruits. Am Rev Respir Dis 1968, 97(6):Supp I-65.

5. Harding SP, Mutton KJ, van der Avoort H, Wermenbol AG: An epidemic of keratoconjunctivitis due to adenovirus type 37 . Eye (London, England) 1988, 2 ( Pt 3):3|4-317.

6. Wood DJ: Adenovirus gastroenteritis. British medical journal (Clinical research ed 1988, 296(66 I7):229-230.

7. Shenk T: Adenoviridae: The Viruses and Their Replication. In Fields Virology Thrid Edition edition. Edited by: B.N. Fields DMKPMH. Philadelphia , Lippincott - Raven Publishers; 1996:2 III-2I 48.

8. Jones MS 2nd, Harrach B, Ganac RD, Gozum MM, Dela Cruz WP, Riedel B, Pan C, Delwart EL, Schnurr DP: New adenovirus species found in a patient presenting with gastroenteritis. Journal of virology 2007, 8 I (I I):5978-5984.

9. de Jong JC, Wigand R, Wadell G, Keller D, Muzerie CJ, Wermenbol AG, Schaap GJ: Adenovirus 37: identification and characterization of a medically important new adenovirus type of subgroup D. Journal of medical virology 1981, 7(2): 105-1 I8.

10. Ariga T, Shimada Y, Shiratori K, Ohgami K, Yamazaki S, Tagawa Y, Kikuchi M, Miyakita Y, Fujita K, Ishiko H, Aoki K, Ohno S: Five new genome types of adenovirus type 37 caused epidemic keratoconjunctivitis in Sapporo, Japan, for more than 10 years. Journal of clinical microbiology 2005, 43(2):726-732.

II. Dhurandhar NV: Contribution of pathogens in human obesity. Drug news \& perspectives 2004, I 7(5):307-3। 3.

12. Fuzznuc: Nucleic Acid Pattern Search [http://bioweb.pas teur.fr/seqanal/interfaces/fuzznuc.html]

13. Purkayastha A, Ditty SE, Su J, McGraw J, Hadfield TL, Tibbetts C, Seto D: Genomic and bioinformatics analysis of HAdV-4, a human adenovirus causing acute respiratory disease: implications for gene therapy and vaccine vector development. Journal of virology 2005, 79(4):2559-2572.

14. Temperley SM, Hay RT: Recognition of the adenovirus type 2 origin of DNA replication by the virally encoded DNA polymerase and preterminal proteins. The EMBO journal 1992, II (2):76I-768.

15. Brudno M, Do CB, Cooper GM, Kim MF, Davydov E, Green ED, Sidow A, Batzoglou S: LAGAN and Multi-LAGAN: efficient tools for large-scale multiple alignment of genomic DNA. Genome research 2003, 13(4):721-73I.

16. Berk AJ, Sharp PA: Structure of the adenovirus 2 early mRNAs. Cell 1978, 14(3):695-7II.

17. Frisch SM, Mymryk JS: Adenovirus-5 EIA: paradox and paradigm. Nature reviews 2002, 3(6):44|-452.

18. Gallimore PH, Turnell AS: Adenovirus EIA: remodelling the host cell, a life or death experience. Oncogene 200I, 20(54):7824-7835.

19. Berk AJ: Recent lessons in gene expression, cell cycle control, and cell biology from adenovirus. Oncogene 2005, 24(52):7673-7685

20. Martin ME, Berk AJ: Adenovirus EIB $55 \mathrm{~K}$ represses $\mathrm{p} 53$ activation in vitro. Journal of virology 1998, 72(4):3146-3154.

21. Yew PR, Berk AJ: Inhibition of p53 transactivation required for transformation by adenovirus early IB protein. Nature 1992, 357(6373):82-85.

22. Horwitz MS: Function of adenovirus E3 proteins and their interactions with immunoregulatory cell proteins. The journal of gene medicine 2004, 6 Suppl I:SI72-83.

23. Windheim M, Hilgendorf A, Burgert HG: Immune evasion by adenovirus E3 proteins: exploitation of intracellular trafficking pathways. Curr Top Microbiol Immunol 2004, 273:29-85.

24. Burgert HG, Blusch JH: Immunomodulatory functions encoded by the E3 transcription unit of adenoviruses. Virus genes 2000 , 2I ( I-2): I 3-25.

25. Deryckere F, Burgert HG: Early region 3 of adenovirus type 19 (subgroup D) encodes an HLA-binding protein distinct from that of subgroups B and C. Journal of virology 1996, 70(5):2832-2841.

26. Leppard KN: E4 gene function in adenovirus, adenovirus vector and adeno-associated virus infections. The Journal of general virology 1997, 78 ( Pt 9):2131-2138.

27. Dobner T, Horikoshi N, Rubenwolf S, Shenk T: Blockage by adenovirus E4orf6 of transcriptional activation by the p53 tumor suppressor. Science 1996, 272(5267): | 470-1473.

28. Moore M, Horikoshi N, Shenk T: Oncogenic potential of the adenovirus E4orf6 protein. Proceedings of the National Academy of Sciences of the United States of America 1996, 93(21): I 1295-I I 30 I.

29. Weiss RS, Lee SS, Prasad BV, Javier RT: Human adenovirus early region 4 open reading frame I genes encode growth-transforming proteins that may be distantly related to dUTP pyrophosphatase enzymes. Journal of virology 1997, $71(3): 1857-1870$

30. Weiss RS, Gold MO, Vogel H, Javier RT: Mutant adenovirus type 9 E4 ORFI genes define three protein regions required for transformation of CREF cells. Journal of virology 1997, 7I(6):4385-4394

3I. Gustin KE, Lutz P, Imperiale MJ: Interaction of the adenovirus LI $52 / 55$-kilodalton protein with the IVa2 gene product during infection. Journal of virology 1996, 70(9):6463-6467.

32. Zhang W, Imperiale MJ: Interaction of the adenovirus IVa2 protein with viral packaging sequences. Journal of virology 2000 , 74(6):2687-2693.

33. Zhang W, Low JA, Christensen JB, Imperiale MJ: Role for the adenovirus IVa2 protein in packaging of viral DNA. Journal of virology 200I, 75(2I): 10446-10454.

34. Alex Dong Li's SpliceSiteFinder [http://www.genet.sick kids.on.ca/ ali/splicesitefinder.html]. Accessed March 4th, 2008

35. Boulanger P, Lemay P, Blair GE, Russell WC: Characterization of adenovirus protein IX. The Journal of general virology 1979, 44(3):783-800.

36. Lutz P, Rosa-Calatrava M, Kedinger C: The product of the adenovirus intermediate gene $I X$ is a transcriptional activator. Journal of virology 1997, 7 I(7):5102-5109.

37. Hasson TB, Ornelles DA, Shenk T: Adenovirus LI 52- and 55 kilodalton proteins are present within assembling virions 
and colocalize with nuclear structures distinct from replication centers. Journal of virology 1992, 66(10):6133-6142.

38. Gustin KE, Imperiale MJ: Encapsidation of viral DNA requires the adenovirus LI 52/55-kilodalton protein. Journal of virology 1998, 72(10):7860-7870.

39. Saban SD, Silvestry M, Nemerow GR, Stewart PL: Visualization of alpha-helices in a 6-angstrom resolution cryoelectron microscopy structure of adenovirus allows refinement of capsid protein assignments. Journal of virology 2006 , 80(24): | 2049-12059.

40. Wickham TJ, Mathias $P$, Cheresh DA, Nemerow GR: Integrins alpha $v$ beta 3 and alpha $v$ beta 5 promote adenovirus internalization but not virus attachment. Cell 1993, 73(2):309-319.

4I. Arnberg N, Kidd AH, Edlund K, Olfat F, Wadell G: Initial interactions of subgenus $D$ adenoviruses with A549 cellular receptors: sialic acid versus alpha(v) integrins. Journal of virology 2000 , 74(16):769I-7693.

42. Chatterjee PK, Vayda ME, Flint SJ: Identification of proteins and protein domains that contact DNA within adenovirus nucleoprotein cores by ultraviolet light crosslinking of oligonucleotides 32P-labelled in vivo. Journal of molecular biology 1986 , I 88(I):23-37.

43. Wodrich H, Guan T, Cingolani G, Von Seggern D, Nemerow G, Gerace L: Switch from capsid protein import to adenovirus assembly by cleavage of nuclear transport signals. The $E M B O$ journal 2003, 22(23):6245-6255

44. Honkavuori KS, Pollard BD, Rodriguez MS, Hay RT, Kemp GD: Dual role of the adenovirus $\mathrm{PVI} C$ terminus as a nuclear localization signal and activator of the viral protease. The Journal of general virology 2004, 85(Pt II):3367-3376.

45. Ebner K, Pinsker W, Lion T: Comparative sequence analysis of the hexon gene in the entire spectrum of human adenovirus serotypes: phylogenetic, taxonomic, and clinical implications. Journal of virology 2005, 79(20): | 2635-I2642.

46. Weber J: Genetic analysis of adenovirus type 2 III. Temperature sensitivity of processing viral proteins. Journal of virology 1976, I7(2):462-47I.

47. Cuesta R, Xi Q, Schneider RJ: Structural basis for competitive inhibition of elF4G-Mnk I interaction by the adenovirus 100 . kilodalton protein. Journal of virology 2004, 78( I 4):7707-77/6.

48. Hayes BW, Telling GC, Myat MM, Williams JF, Flint SJ: The adenovirus L4 100-kilodalton protein is necessary for efficient translation of viral late mRNA species. Journal of virology 1990 , 64(6):2732-2742.

49. Hong SS, Szolajska E, Schoehn G, Franqueville L, Myhre S, Lindholm L, Ruigrok RW, Boulanger P, Chroboczek J: The I00K-chaperone protein from adenovirus serotype 2 (Subgroup C) assists in trimerization and nuclear localization of hexons from subgroups C and B adenoviruses. Journal of molecular biology 2005, 352(I): $125-138$

50. Liu GQ, Babiss LE, Volkert FC, Young CS, Ginsberg HS: A thermolabile mutant of adenovirus 5 resulting from a substitution mutation in the protein VIII gene. Journal of virology 1985, 53(3):920-925.

51. Ostapchuk P, Anderson ME, Chandrasekhar S, Hearing P: The L4 22kilodalton protein plays a role in packaging of the adenovirus genome. Journal of virology 2006, 80(14):6973-698I.

52. Arnberg N, Mei Y, Wadell G: Fiber genes of adenoviruses with tropism for the eye and the genital tract. Virology 1997 227(I):239-244

53. Nicol CG, Graham D, Miller WH, White SJ, Smith TA, Nicklin SA, Stevenson SC, Baker AH: Effect of adenovirus serotype 5 fiber and penton modifications on in vivo tropism in rats. Mol Ther 2004, I0(2):344-354.

54. Smith TA, Idamakanti N, Rollence ML, Marshall-Neff J, Kim J, Mulgrew K, Nemerow GR, Kaleko M, Stevenson SC: Adenovirus serotype 5 fiber shaft influences in vivo gene transfer in mice. Human gene therapy 2003/06/14 edition. 2003, 14(8):777-787.

55. Smith TA, Idamakanti N, Marshall-Neff J, Rollence ML, Wright $P$, Kaloss M, King L, Mech C, Dinges L, Iverson WO, Sherer AD, Markovits JE, Lyons RM, Kaleko M, Stevenson SC: Receptor interactions involved in adenoviral-mediated gene delivery after systemic administration in non-human primates. Human gene therapy 2003/I I/25 edition. 2003, I4(I7):1595-1604.
56. O'Malley RP, Mariano TM, Siekierka J, Mathews MB: A mechanism for the control of protein synthesis by adenovirus VA RNAI. Cell 1986, 44(3):391-400.

57. Liao HJ, Kobayashi R, Mathews MB: Activities of adenovirus virusassociated RNAs: purification and characterization of RNA binding proteins. Proceedings of the National Academy of Sciences of the United States of America 1998, 95(I5):85I4-85I9.

58. Andersson MG, Haasnoot PC, Xu N, Berenjian S, Berkhout B, Akusjarvi G: Suppression of RNA interference by adenovirus virusassociated RNA. Journal of virology 2005, 79(15):9556-9565.

59. Kidd $A H$, Garwicz D, Oberg M: Human and simian adenoviruses: phylogenetic inferences from analysis of VA RNA genes. Virology 1995, 207(I):32-45

60. Pearson WR: Flexible sequence similarity searching with the FASTA3 program package. Methods Mol Biol 2000, I32:|85-2| 9 .

6I. Hiller K, Schobert M, Hundertmark C, Jahn D, Munch R: JVirGel: Calculation of virtual two-dimensional protein gels. Nucleic acids research 2003, 3 I ( I 3):3862-3865.

62. JVirGel v2.0 [http://www.jvirgel.de/

63. Patrickios CS, Yamasaki EN: Polypeptide amino acid composition and isoelectric point. II. Comparison between experiment and theory. Analytical biochemistry 1995, 23 I(I):82-91.

64. Skoog B, Wichman A: Calculation of the isoelectric points of polypeptieds from the amino acid composition. Trends Anal Chem 1986:82-83.

65. Tabrizi SN, Ling AE, Bradshaw CS, Fairley CK, Garland SM: Human adenoviruses types associated with non-gonococcal urethritis. Sexual health 2007, 4(I):4I-44.

66. Davison AJ, Benko M, Harrach B: Genetic content and evolution of adenoviruses. The Journal of general virology 2003, 84(Pt I I):2895-2908

67. Natarajan K, Shepard LA, Chodosh J: The use of DNA array technology in studies of ocular viral pathogenesis. DNA and cell biology 2002, 2 I (5-6):483-490.

68. Engelmann I, Madisch I, Pommer H, Heim A: An outbreak of epidemic keratoconjunctivitis caused by a new intermediate adenovirus $22 / \mathrm{H} 8$ identified by molecular typing. Clin Infect Dis 2006, 43(7):e64-6

69. Lauer KP, Llorente I, Blair E, Seto J, Krasnov V, Purkayastha A, Ditty SE, Hadfield TL, Buck C, Tibbetts C, Seto D: Natural variation among human adenoviruses: genome sequence and annotation of human adenovirus serotype I. The Journal of general virology 2004, 85(Pt 9):26|5-2625

70. Rozen S, Skaletsky H: Primer3 on the WWW for general users and for biologist programmers. Methods Mol Biol 2000, 132:365-386

7I. Gordon D, Abajian C, Green P: Consed: a graphical tool for sequence finishing. Genome research 1998, 8(3): 195-202.

72. Ewing B, Hillier L, WendI MC, Green P: Base-calling of automated sequencer traces using phred. I. Accuracy assessment. Genome research 1998, 8(3): 175-185.

73. Ewing B, Green P: Base-calling of automated sequencer traces using phred. II. Error probabilities. Genome research 1998, 8(3): $186-194$

74. JCVI Annotation Service [http://www.jcvi.org/cms/research/ projects/annotation-service/overview/]

75. Manatee [http://manatee.sourceforge.net/]

76. GeneMark [http://exon.gatech.edu/GeneMark/]

77. NCBI's ORF Finder (Open Reading Frame Finder) [http:// www.ncbi.nlm.nih.gov/gorf/gorf.html]

78. VISTA [http://genome.lbl.gov/vista/index.shtml]

79. EBI Tools:: FASTA and SSEARCH similarity searching against protein databases [http://www.ebi.ac.uk/fasta33/]

80. BLAST: Basic Local Alignment and Search Tool [http:// www.ncbi.nlm.nih.gov/BLAST/Blast.cgi]

81. Kumar S, Tamura K, Nei M: MEGA3: Integrated software for Molecular Evolutionary Genetics Analysis and sequence alignment. Briefings in bioinformatics 2004, 5(2): 150-163. 